\title{
Metaiodobenzylgutanidine scintigraphy of cardiac sympathetic innervation
}

\section{Anna Teresińska}

Department of Nuclear Medicine, The Cardinal Stefan Wyszyński Institute of Cardiology, Warsaw, Poland

\section{[Received 18 | 2012; Accepted 2 || 2012]}

\begin{abstract}
Metaiodobenzylguanidine (MIBG), a noradrenaline analogue, labelled with radioactive iodine $\mathrm{I}-123$ is used for assessing pre-synaptic activity of the myocardial adrenergic system. The paper describes the mechanism of myocardial I-123-MIBG uptake and the relevant examination methods. It also refers to the necessity of standardising acquisition methods and assessment criteria in order to improve comparability of results, especially numerical results, originating from various countries and centres. Although first introduced as a cardiological diagnostic procedure 30 years ago, I-123-MIBG myocardial scintigraphy continues to be mostly applied as a research method, even though its diagnostic and/or prognostic importance, especially in the case of heart failure, has been widely reported. The recently published results of two extensive retrospective works and a major prospective, multi-centre international ADMIRE-HF study confirmed that I-123-MIBG scintigraphy has a high independent prognostic value in evaluating patients with heart failure. Abnormal myocardial I-123-MIBG uptake can also be indicative of higher risk of ventricular arrhythmias and sudden cardiac death in patients with heart failure. I-123-MIBG myocardial scintigraphy also seems to have considerable potential for the detection and differentiation of neurodegenerative diseases.
\end{abstract}

Key words: cardiac sympathetic innervation, scintigraphy, iodine-123 metaiodobenzylguanidine, MIBG

Nuclear Med Rev 2012; 15, 1: 61-70

Correspondence to: Anna Teresińska

Department of Nuclear Medicine

The Cardinal Stefan Wyszyński Institute of Cardiology

Alpejska 42, 04-628 Warsaw, Poland

Tel: +4822343 4281, fax: +48223434513

e-mail: ateresinska@ikard.pl

\section{Introduction}

The autonomic nervous system consists of the adrenergic (sympathetic) system with stimulating action - and noradrenaline as the main transmitter, and the cholinergic (parasympathetic) system with inhibiting action - and acetylcholine as the main transmitter. The imaging of the cardiac cholinergic system is very limited due to the low density of parasympathetic nerves in the ventricular muscles and the lack of appropriate analogues of acetylcholine, which undergoes rapid decomposition in the presynaptic nerve terminals, suitable for radioisotope examinations. However, the adrenergic fibres are abundant in the heart — they run in the epicardium along the coronary arteries and penetrate the myocardium similarly as the smaller coronary vessels; they are present in the ventricles (in particular at the base thereof) and to a significant extent are more concentrated in the atria.

Metaiodobenzylguanidine (MIBG) is a chemical analogue of noradrenaline - its uptake and storage mechanisms in the adrenergic nerve terminals are similar to those of noradrenaline, while it hardly undergoes metabolism and remains unchanged for a longer period of time in the nerve terminals - therefore, when labelled with radioactive iodine, it is considered a pharmaceutical suitable for the assessment of the presynaptic portion of the cardiac adrenergic innervation [1-6].

As the radiation energy $(159 \mathrm{keV}$ ) of radioactive iodine $\mathrm{I}-123$ is comparable to that of technetium Tc-99m (thus it is possible to use low-energy collimators and acquire relatively high-resolution scintigraphic images) and it has comparatively short half-life $(13.3 \mathrm{~h})$, it currently seems to be the best one of the available iodine radioisotopes for MIBG labelling.

The I-123-MIBG radiotracer, used for assessing the presynaptic part of the adrenergic system, is the most extensively researched and practically the only radiotracer available in the classical scintigraphy of the cardiac autonomic nervous system. This radiotracer was first used for the assessment of a human heart by Kline et al. in 1981 [1].

Other imaging agents include noradrenaline analogues labelled with positron radiotracers for PET scintigraphy (hydroxyephedrine, epinephrine and phenylephrine labelled with C-11 [7] and a completely new LMl1195 radiotracer for labelling with F-18 which has a longer half-life [8])

The research is also underway into the labelling of post-synaptic receptors which transmit signals from the adrenergic system to 
the myocardial tissue, regulating the chronotropic, inotropic and dromotropic effects within the myocardium. There are reports of clinical applications of the CGP12177 positron radiotracer labelled with $\mathrm{C}-11$ - non-selective hydrophilic compound binding to beta-receptors - to demonstrate their myocardial density in diagnosing [9] and prognosing [10] based on PET scintigraphy.

\section{Description of MIBG uptake, examination methods and interpretation of results}

\section{Mechanism of the myocardial MIBG uptake}

Following intravenous administration, MIBG diffuses from the vascular to the intracellular space, and from there it is actively transported, similarly as noradrenaline, to the adrenergic terminals or a small fraction of MIBG diffuses (to neurons, muscle cells, the vascular space)

The first mechanism (so-called uptake-1, energy-dependent) is the prevailing mechanism. From the synaptic cleft, MIBG undergoes active uptake at the nerve terminal which is facilitated by the specific noradrenaline monoamine transporter (NET) protein and most of the radiotracer, by means of another active transport process facilitated by the specific vesicular monoamine transporter (VMAT) protein, is stored in noradrenaline-storing vesicles in the neuron's terminal. Uptake-1 becomes saturated. Nerve stimulation induces the secretion of noradrenaline and MIBG: vesicles combine with the neuron's membrane and secrete their content to the synaptic space by exocytosis. Metaiodobenzylguanidine is re-transported to the nerve terminal by the uptake-1 mechanism or diffuses (into the vascular space and conceivably to neurons, and also to myocytes in animals). Unlike the noradrenaline fraction which is not stored in the vesicles, but metabolised in the cytosol of the nerve terminal [by monoamine oxidase (MAO)], MIBG is not catabolised — therefore the extravesicular concentration of MIBG is larger than that of noradrenaline. Metaiodobenzylguanidine can diffuse from the cytosol of the nerve terminal into the intracellular space.

The mechanism eliminating noradrenaline into the extraneural tissue (sometimes called uptake-2) is probably based on passive diffusion. Uptake-2 has no saturation point. It was shown using animal models that the extraneural uptake-2 of MIBG can reach a significant level. However, it is not the same in humans - no MIBG uptake is observed in the transplanted (and initially fully de-innervated) heart until at least 4 months after the transplantation. In contrast to noradrenaline, MIBG in the cardiomyocyte cytosol is not catabolised by catechol-O-methyltransferase (COMT).

The compound exhibits low affinity to post-synaptic adrenergic receptors, and for that reason - as opposed to noradrenaline — has a highly insignificant pharmacological effect.

It was demonstrated that the level of myocardial MIBG uptake is correlated with the myocardial noradrenaline level [13] and it is explicitly dependent on the presence of non-damaged sympathetic nerve fibres [14-17].

\section{MIBG pharmacokinetics and pharmacodynamics}

The dynamics of myocardial MIBG accumulation can be described as follows:

- after intravenous administration, myocardial MIBG uptake follows immediately and achieves a stable level after 2-3 mi- nutes [60], after 60 minutes it reaches about $1 \%$ of the administered dosage [1];

- intravesicular myocardial accumulation of I-123-MIBG is relatively constant starting from the $5^{\text {th }}$ minute after administration, while extravesicular myocardial accumulation decreases to a significant extent between the $5^{\text {th }}$ minute and $6^{\text {th }}$ hour after the administration; the intravesicular percentage of the total myocardial accumulation achieves a constant (and maximal) value equal to $50 \%$ within 4 hours after the administration; hence it is considered that images acquired about 4 hours after the injection of the tracer would best reflect the neuronal MIBG uptake in the heart [19].

MIBG pharmacokinetics and pharmacodynamics in the human body is as follows:

- I-123-MIBG blood level decreases quite rapidly: about 10\% of the injected dosage remains in blood after 5 minutes from the administration, about $5 \%$ after 15 minutes, and about $2 \%$ after 1 hour [1];

- the highest MIBG fraction is accumulated in the liver (about 0.33 of the administered dosage), the greatest uptake is observed in the lungs (about 0.03), the heart (0.008), the spleen (about 0.006) and in the salivary glands (about 0.004);

- tracer uptake in the liver is always greater compared to the heart: the ratio of the myocardial-to-hepatic uptake is about 0.7 after 15 minutes and remains at 0.3-0.6 during the next 24 hours $[1,11,20]$;

- tracer uptake in the lungs is always smaller compared to the myocardium: the ratio of the myocardial-to-pulmonary uptake is about 1.2 after 15 minutes, 1.4 after 2 hours and 2.9 after 24 hours [1, 11];

- I-123-MIBG is excreted from the body through the kidneys - $85-90 \%$ as an unchanged compound (scarcely any amount of the tracer is metabolised in living organisms) and about $10 \%$ as a de-iodinated compound; $55 \%$ of MIBG is excreted within 24 hours after the injection and $90 \%$ after 4 days.

\section{Impact of medication on MIBG accumulation and preparation of patients for examination}

A number of drugs are known or can be assumed to have an impact on the MIBG uptake and/or storage [21-23, 24]. Patients should be taken off such medication (if possible) for a sufficiently long period of time before the examination. If it is impossible to discontinue medication, at least its possible impact on the acquired images of the adrenergic system should be taken into consideration.

There are several mechanisms which affect - separately or jointly after the administration of specific drugs — the MIBG storage process (approved names of the drugs which have been demonstrated to influence the MIBG uptake in specific organs are given in brackets):

- inhibited active uptake (cocaine, tricyclic antidepressants, labetalol);

— inhibited active transport to vesicles (reserpine);

- competition for vesicular transport (guanethidine);

- secretion of the vesicular content (sympathomimetic drugs, labetalol, reserpine);

- other non-explained mechanisms. 
Table 1. Recommended acquisition parameters for cardiac I-123-MIBG studies [25]

\begin{tabular}{ll}
\hline Parameter & Value/Description \\
\hline Energy window: & $20 \%$ symmetrically around $159 \mathrm{keV}$ \\
Collimators: & Low energy high resolution (LEHR) or medium energy (ME) \\
Planar imaging: & Anterior \\
- projection: & $128 \times 128$ or $256 \times 256$ \\
- matrix: & 10 min and $4 \mathrm{~h}$ \\
- starting time p.i.: & 10 min \\
- length of each acquisition: & \\
SPECT imaging: & Single or dual heads (in a 90 or "L" configuration) \\
- detectors: & $180^{\circ}$ (starting at $45^{\circ}$ right anterior oblique projection and proceeding anticlockwise to the $45^{\circ}$ left \\
- rotation: & posterior oblique projection) \\
& 64 \\
- number of projections: & $20 / 25 \mathrm{~s}$ \\
- time/projection: & $\leq 25$ min \\
\hline overall acquisition time: &
\end{tabular}

It is also recommended that patients should refrain from consuming foods containing vanilla and catecholamine-like ingredients (e.g. chocolate, blue cheese) as some of them might interfere with the MIBG uptake.

MIBG is usually administered after the uptake of free iodine- 123 by the thyroid is blocked. The thyroid can be blocked by administering potassium or sodium perchlorate (400-500 mg) or Lugol's iodine (equivalent of $130 \mathrm{mg}$ of potassium iodide) at least 30 minutes before the MIBG injection [24, 25].

Patients should drink plenty of liquids and frequently urinate for the first 48 hours after the administration of MIBG to accelerate the excretion of the radiopharmaceutical from their bodies and reduce the exposure of the bladder and the whole body to radiation.

\section{MIBG examinations of the myocardial adrenergic system}

Acquisition parameters recommended for cardiac I-123-MIBG studies are shown in Table 1.

Normal-weight adult patients are administered a dosage of 111-370 MBq (3-10 $\mathrm{mCi}$ ) of the radiopharmaceutical [25].

The effective dosage received by an adult patient after the administration of I-123-MIBG is $0.013 \mathrm{mSv} / \mathrm{MBq}$ (i.e. a standard patient administered $370 \mathrm{MBq}$ of the radiopharmaceutical receives a dosage of $4.81 \mathrm{mSv}$ ).

MIBG should be administered intravenously very slowly (for at least 1-2 minutes according to [25], and for at least 5 minutes according to [24]).

During the acquisition of scintigraphic images a 15- or 20-percent energy window is used, positioned symmetrically around the iodine-123 photopeak (159 keV).

Planar images of the chest are registered (anterior projection, $128 \times 128$ matrix, acquisition time -10 minutes); early images are registered 15 minutes, and late images 4 hours after the administration of the radiopharmaceutical. As the planar technique is known to have limitations (overlapping images of extra-cardiac structures and myocardium as well as no possibility of segmental analysis), additional SPECT images ( $64 \times 64$ matrix $)$ can be registered immediately after the registration of planar images.
Medium-energy collimators are preferred in the case of iodine-123 as in addition to the primary emission of photons at $159 \mathrm{keV}$, iodine-123 emits also about $3 \%$ of photons at $>400$ $\mathrm{keV}$ which leads to the penetration of the septa in low-energy high-resolution (LEHR) collimators and causes a high contribution of scattered radiation to the energy window around $159 \mathrm{keV}$. Medium-energy collimators enable better diagnostic accuracy of I-123 examinations compared to low-energy collimators. However, so far nearly every report relating to the quantitative assessment of the myocardial I-123-MIBG uptake in patients with heart failure (including all 96 centres registered in the most extensive prospective international study - ADMIRE-HF [26], 5/6 centres included in the retrospective multi-centre European study [27] and probably $17 / 18$ works underlying a meta-analysis of the best studies [28]) was based on the acquisition of images with low-energy collimators. The numerical data gathered in these studies cannot be used for interpreting results obtained with medium-energy collimators.

\section{Assessment of the global myocardial MIBG uptake based on the planar examination}

The methods underlying the assessment of MIBG myocardial planar examinations are based on a semi-quantitative measurement of the global radiotracer uptake in the heart after $15 \mathrm{mi}-$ nutes and/or 4 hours from its administration and/or the washout rate of the tracer from the heart between the $15^{\text {th }}$ minute and $4^{\text {th }}$ hour from its administration.

The global uptake of I-123-MIBG is most often expressed as the $\mathrm{H} / \mathrm{M}$ ratio, i.e. the ratio of the average number of counts within the heart to the average number of counts within the mediastinum (i.e. the background) after the examined area of the heart and the upper part of the sternum are mapped onto the scintigraphic planar image in anterior projection [25] (Figure 1). The H/M ratio after 15 minutes $\left(H / M_{15 m}\right)$ most probably reflects the continuity of pre-synaptic nerve terminals and the functioning of uptake-1. The $\mathrm{H} / \mathrm{M}$ ratio after 4 hours $\left(\mathrm{H} / \mathrm{M}_{4 \mathrm{~h}}\right)$ enables integrating information about the neuronal function, starting from the MIBG uptake, through storage, up to the ejection from the storing vesicles in the nerve terminals. It was demonstrated that the $\mathrm{H} / \mathrm{M}$ ratio after 


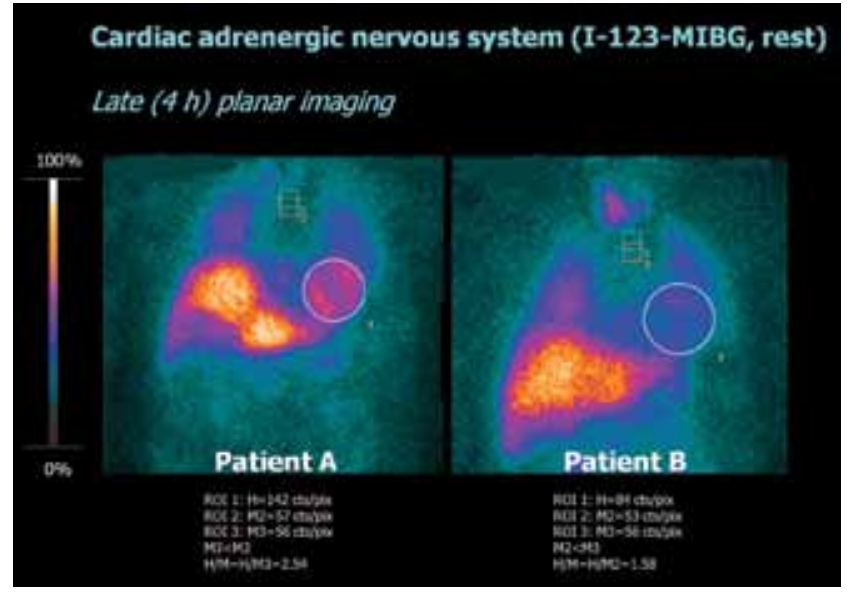

Figure 1. Planar anterior I-123-MIBG images obtained 4 hours after radiopharmaceutical injection in two patients with ischaemic heart failure. The counts per pixel within a circular ROI $1(\mathrm{H})$ correspond to the global radioactivity accumulated in the heart. The counts per pixel within the square $\mathrm{ROI} 2$ or $\mathrm{ROI} 3$ ( $\mathrm{M}$, from the region with lower value) correspond to the non-specific radioactivity accumulated in the upper mediastinum. The heart-to-mediastinum ratio $(\mathrm{H} / \mathrm{M})$ is used as a measure of myocardial uptake of MIBG. A. Patient with high MIBG myocardial uptake $\left(H / M_{4 h}=2.54\right)$. B. Patient with lower MIBG uptake $\left(H / M_{4 h}=1.58\right)$ and a higher risk of major cardiac events

4 hours is correlated with the total myocardial MIBG uptake referred to as $\%$ of the uptake of the administered dosage $(r=0.72$, $\mathrm{p}<0.0001$ ) [29]. Furthermore the $\mathrm{H} / \mathrm{M}$ ratio is correlated with the myocardial noradrenaline level $(r=0.63)$ [13]. Thus, the $\mathrm{H} / \mathrm{M}$ ratio is a measure of the global myocardial MIBG uptake and can be used as a practical rate of uptake [29-31].

The washout rate (WR) of MIBG from the heart is most often expressed as a percentage difference between the average number of counts within the heart after 15 minutes and 4 hours and the average number of counts within the heart after 15 minutes. However, there is a wide variety of calculation methods - with or without standardisation of $\mathrm{H}$-to-M counts, with or without background correction by subtracting $\mathrm{M}$ counts from $\mathrm{H}$ counts, with or without correction for radioisotope decay in time [25, 32, 33]. The washout rate of MIBG from the heart indicates the excitation level of the adrenergic system (greater activity of the myocardial adrenergic system is related to the high WR value and low MIBG uptake in late images). Nonetheless, there are doubts about the interpretation of the washout rate - most probably it represents several mechanisms (at least vesicular exocytosis and extravesicular diffusion in the nerve terminals). Greater WR values were described in a number of cardiological diseases - but the mechanisms responsible for the phenomenon have not been fully explained.

Most often the $\mathrm{H} / \mathrm{M}$ ratio, in particular after 4 hours from the MIBG administration, is considered to be the most important factor in the global assessment of the myocardial adrenergic system - also on account of the larger number of studies in which this parameter was tested. However, it has not been unambiguously determined whether any single parameter - $\mathrm{H} / \mathrm{M}$ or WR - or their combination should be taken into consideration in the prognostic process.

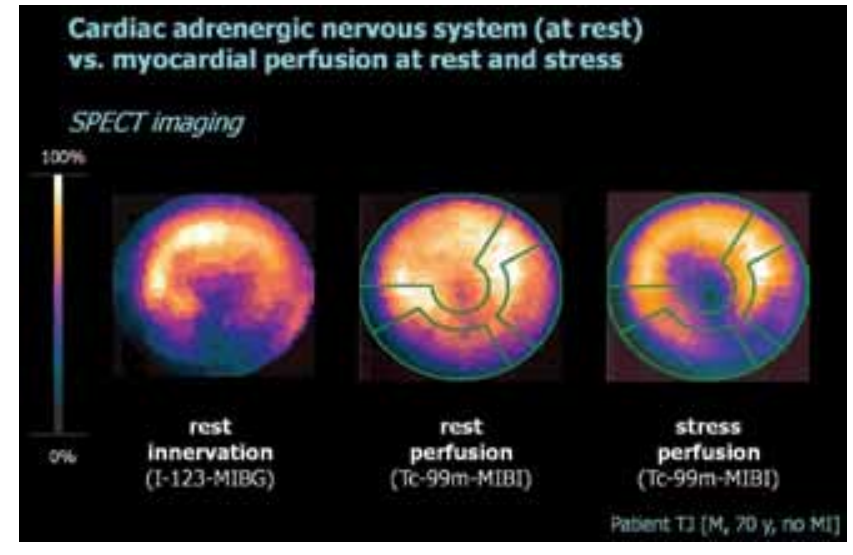

Figure 2. Comparison of the images of regional adrenergic innervation (at rest) and perfusion (at rest and under stress) of the left ventricular myocardium in SPECT studies. From each study, the summary uptake of the radiotracer ('bull's-eye' map) is presented. I-123-MIBG uptake deficit evaluated at rest is much bigger than Tc-99m-MIBI uptake deficit at rest; rest image of the innervation is more corresponding to perfusion image in stress conditions

\section{SPECT-based assessment of the regional myocardial MIBG uptake}

The papers known from the literature on the SPECT-based examination of "normal" myocardial I-123-MIBG uptake, performed within several hours after the administration of radiotracer, were related to the relative assessment of the radiotracer distribution in specific regions of the left-ventricular muscle and the determination of the correlation between the distribution and the patient's sex and/or age [34-38]. Normal I-123-MIBG uptake in the left-ventricular muscle is non-uniform and depends on the patient's age and probably sex. It also seems that the normal relative uptake in specific regions is comparable to the relative uptake of perfusion tracers in the muscle: the greatest number of counts is observed above the anterolateral wall, the lowest number above the lower wall (especially in men). Reduced number of counts can be present in the apex. The nerves of the cardiac adrenergic system are more sensitive to ischaemia than cardiomyocytes. Therefore, the defects observed in MIBG rest images are bigger (more extensive and more intense) than the rest perfusion defects and more often are similar to stress-induced ischaemic perfusion defects (Figure 2).

It should be noted that if the primary objective of the MIBG uptake assessment is not to diagnose the myocardial adrenergic innervation in a given population (compared to normal results), but the evaluation of any expected regional changes in the innervation in relation to the initial condition of a given patient or of any differences in the regional innervation between well-defined groups of patients, then it is not critically necessary to have maps of normal uptake levels.

The quality of I-123-MIBG SPECT can be much lower than the quality of SPECT-based myocardial perfusion with technetium tracers. As many as $16 \%$ of the I-123-MIBG SPECT examinations carried out by our team [39] were non-diagnostic studies due to the very low uptake level within the myocardium and/or very high radioactivity in non-myocardial structures (liver, bowels, lungs), the images of which were not separated from myocardial images. Also Gill et al. [34] reported that the artefacts on the inferior left ventricu- 
lar wall — due to the very high MIBG uptake in the liver or spleen in SPECT images registered within 3 hours after the administration of the tracer - were so frequent that the authors decided to discontinue the assessment of radiotracer distribution in this wall.

\section{Necessity of standardisation of MIBG examinations}

During their retrospective assessment of the studies relating to the myocardial adrenergic system, Agostini, Verberne and Jacobson determined large differences in the acquisition methods applied in Europe, which could affect the resulting parameters of the global MIBG uptake [27]. Their efforts to standardise the technical aspects of the procedure (and thus to increase the prospect that it is accepted as a clinical tool for patients with heart failure) [32, 33] resulted in a proposal for standardisation of I-123-MIBG imaging of the myocardial adrenergic system, announced by the Cardiovascular Committee of the European Association of Nuclear Medicine and the European Council of Nuclear Cardiology [25]. It should contribute to reducing differences between results, including numerical results, originating from various countries and centres [40].

\section{Clinical applications of I-123-MIBG myocardial examinations}

Although first introduced as a cardiac diagnostic procedure in the early 1980s, I-123-MIBG myocardial scintigraphic methods continue to be mostly applied in scientific research (Table 2). Their advantage over the existing diagnostic methods has not been sufficiently documented (lack of multi-centre randomised studies with large groups of patients), even though there are numerous reports of the diagnostic and/or prognostic importance of this examination, especially in the case of heart failure. The testing results of MIBG-based examinations have been described for a variety of other heart diseases, including: primary arrhythmias [41-43], myocardial ischaemia with infarction [14-17] or without [44-48], in the assessment of the development of the innervation system after heart transplant $[11,12,49]$, in the assessment of cardiotoxicity of anticancer chemotherapy [50-52]. There are also numerous reports showing that the I-123-MIBG imaging enables effective monitoring of the effects of conventional pharmacological therapies; images of MIBG uptake are improved after therapy with beta-blockers and often associated with reduced volume of the ventricles, better LVEF and reduced symptoms of heart failure; carvedilol has been most extensively researched in this respect (the recent randomised multi-centre double-blind placebo-controlled study was the French study described by Cohen-Solal in 2005 [53]); also other approved drugs which do not directly affect the myocardial adrenergic functions, such as ACE inhibitors, ARB blockers, spironolactone or amiodarone, have also been shown to improve MIBG uptake. MIBG-based myocardial examinations have also been applied in cardiomyopathies [13, 54-58], myocarditis [59], and also in diabetic neuropathy [60-63, MIBG examinations will perhaps enable identifying high-risk patients - Nagamachi observed patients with type 2 diabetes, with no cardiological symptoms, for an average period of 7 years; $\mathrm{H} / \mathrm{M}_{4 \mathrm{~h}}<1.7$ was an independent predictor of general mortality rate] and in neurological diseases (mainly in Parkinson's disease). Furthermore I-123-MIBG was used in studies of the impact of transmyocardial laser revascularisation (TMLR) on live tissue, which
Table 2. Clinical applications of I-123-MIBG myocardial examinations

Heart diseases

Heart failure

Primary arrhythmias

Coronary artery disease with infarction

Myocardial ischaemia without infarction

Assessment of the development of the innervation system after heart transplantation

Assessment of cardiotoxicity of anticancer chemotherapy

Monitoring of the effects of pharmacological therapies

Cardiomyopathies

Myocarditis

Diabetic neuropathy

Churg-Strauss syndrome

Neurological diseases

Diseases with Lewy bodies:

- Parkinson's disease (PD)

- Dementia with Lewy bodies (DLB)

Parkinson's syndromes

Other types of dementia [mainly Alzheimer's disease (AD)]

showed that a damage of the myocardial adrenergic system can be responsible for the significant clinical improvement generally observed at an early stage after TMLR, however, in the light of reinnervation, it is not responsible for the improvement covering a period of several years [64-67].

Most recently MIBG imaging was applied for the assessment of cardiac involvement and prediction of cardiac events in patients with Churg-Strauss syndrome (a rare variety of systemic vasculitis, often involving myocardial vessels) [68].

Below are discussed results of the studies relating to some selected clinical situations which have been described in a number of publications and new original works published over the past 2 years, which shows the continuous/increasing interest in I-123-MIBG myocardial examinations.

\section{I-123-MIBG examinations in heart failure}

The clinical applications of I-123-MIBG in cardiology have been most extensively examined in patients with heart failure.

Heart failure (HF) is a condition with clinical symptoms of impaired heart performance - patients experience shortness of breath at rest or during exertion and/or fatigue, have symptoms of fluid retention, such as stasis in the pulmonary circulation or oedema around ankles, and the objective data suggest irregularities in the heart structure and functions at rest. The incidence of $\mathrm{HF}$ in Europe varies from 2 to 3\%, but it increases rapidly around the age of 75 (10-20\% in the age group of 70-80 years). Coronary artery disease is still the most frequent cause of myocardial disease and it is estimated to be a causative factor in about $70 \%$ of $\mathrm{HF}$ patients. The general prognosis in HF is poor; on average $50 \%$ of HF patients die within 4 years of the diagnosis [69].

The adrenergic nervous system plays one of the major roles in HF pathophysiology. The primary changes in HF are characte- 
rised by the increased activity of the adrenergic system (however, the precise cause of sympathetic activation in HF is not known). Initially, the increased sympathetic activation (greater conductivity in the sympathetic nerves) is a compensating mechanism: increased noradrenaline (NE) secretion from the neuron terminal of the myocardial adrenergic system causes a greater amount of $\mathrm{NE}$ in the synaptic cleft and increases the myocardial contractility and heart rate. A small fraction of NE passes from the cleft to the blood circulation and thus increases the NE level in blood plasma, and the remaining NE is transported by NET back to the nerve terminal; increased NE secretion is usually associated with reduced NE reuptake to the nerve terminal due to the reduced sensitivity of the NET transporter. In patients with chronic HF the greater activity of the myocardial adrenergic system has adverse effects - increased NE concentration in the synaptic cleft decreases the sensitivity of post-synaptic adrenergic beta- 1 receptors on the cardiomyocyte membrane, reduces the activation conductivity and contributes to the impairment of heart functions. These changes in the activity of the myocardial adrenergic system are also relevant to the generation of ventricular arrhythmias and sudden cardiac death in HF patients.

As the reduced NE reuptake contributes to the low MIBG concentration in neurons (reduced $\mathrm{H} / \mathrm{M}$ ratio) and the increased NE secretion causes greater MIBG washout (greater WR value), an assessment of the activity of the adrenergic system based on MIBG as well as $\mathrm{H} / \mathrm{M}$ and WR imaging parameters is used for obtaining prognostic information in the case of HF patients.

The study of Merlet et al., published in 1992, was the first report showing that the myocardial MIBG uptake is a strong predictive factor for cardiac events in the case of patients with chronic HF [31]. Low H/M value was determined to be the best independent predictor of the death rate, better than low EF value: after 20 months the survival rate was $10 \%$ in the case of patients with $\mathrm{H} / \mathrm{M}<1.22$, and $30 \%$ in the case of patients with $\mathrm{EF}<20 \%$.

It has been shown in a number of single-centre studies that the myocardial MIBG uptake has a prognostic value for HF patients. Recently Agostini et al. have carried out a retrospective review and an important prospective quantitative re-analysis of the studies conducted over the past 10 years in 6 European centres with a view to verifying these observations based on the rigorous methodology of clinical studies [27]. Their study focused on the analysis of the $\mathrm{H} / \mathrm{M}$ ratio after four hours $\left(\mathrm{H} / \mathrm{M}_{4 h}\right)$, and more specifically, on account of considerable non-uniformity of the methods applied in each centre, after 2-7 hours from the administration of a radiotracer - in HF patients, Class II-IV according to the NYHA classification. (Early scans were only available for $28 \%$ of the patients, and SPECT results for $7 \%$ of the patients.) 290 patients with EF $<50 \%$ (including $42 \%$ of the patients with ischaemic heart failure) were evaluated in the study. Major cardiac events (MCEs) defined as: cardiac death (infarction, HF progression, SCD), transplantation, potentially life-threatening arrhythmia, were observed over a period of 24 months. The images acquired during blind studies were subject to standardised assessment. In the MCE group, the average $\mathrm{H} / \mathrm{M}_{4 \mathrm{~h}}$ ratio was $1.51 \pm 0.3$, and in the non-MCE group $-1.97 \pm 0.54(p<0.0001)$. The optimised cut-off threshold for the $\mathrm{H} / \mathrm{M}_{4 \mathrm{~h}}$ ratio was defined as 1.75 ; 2-year survival rate for non-MCE patients was $62 \%$ with lower values, and $95 \%$ with values $\geq 1.75$. Additionally, the non-MCE survival rate was $52 \%$ for the high-risk $\mathrm{H} / \mathrm{M}_{4 \mathrm{~h}}$ value $\leq 1.45$, and $98 \%$ for the low-risk $\mathrm{H} / \mathrm{M}_{4 \mathrm{~h}}$ value $\geq 2.18$. It was shown that the $\mathrm{H} / \mathrm{M}_{4 \mathrm{~h}}$ parameter was the strongest independent predictor of the risk of MCE occurrence; the EF parameter ranked second, a weaker predictor. The assessment of interactions between $\mathrm{EF}$ and $\mathrm{H} / \mathrm{M}_{4 \mathrm{~h}}$ values showed that the prognostic value of the $\mathrm{H} / \mathrm{M}_{4 \mathrm{~h}}$ parameter was evident in the population of patients with intensified dysfunction of the left ventricle ( $\mathrm{EF} \leq 35 \%)$.

The application of the methodology used in clinical studies based on a retrospective analysis of MIBG uptake images confirmed the previously reported prognostic value of this method for HF patients, including a possibility of determining the numerical threshold indicating low risk of cardiac death, necessary transplantation or serious ventricular arrhythmias [27].

At the same time as Agostini et al. conducted their analysis, another study was published - a meta-analysis of 18 studies with a total of 1755 patients with heart failure (including $31 \%$ with ischaemic HF) — carried out by Verberne et al. [28]. The objective of the systematic review was to provide a critical assessment of the existing facts relating to the prognostic value of semi-quantitative myocardial I-123-MIBG uptake parameters $\left(H / M_{15 m}, H / M_{4 h}, W R\right)$ for HF patients. The occurrence of cardiac death and cardiac events (cardiac death, stroke, transplantation, hospitalisation due to HF progression) was assessed. It was shown that patients with reduced $\mathrm{H} / \mathrm{M}_{4 \mathrm{~h}}$ ratio or greater WR value have a considerably worse prognosis.

Recently there were published the results of the first large (961 patients with $\mathrm{EF} \leq 35 \%$ and assigned to Class II-III according to the NYHA classification) multi-centre international prospective study conducted by Jacobson et al. (ADMIRE-HF Study [26]) which included 96 centres from North America and Europe. The study focused on the analysis of the $\mathrm{H} / \mathrm{M}_{4 \mathrm{~h}}$ parameter. Control observations covered a period of 2 years. The endpoint was determined as the occurrence of a cardiac event defined as a progression of NYHA class or occurrence of potentially life-threatening arrhythmia or cardiac death. It was compared to $\mathrm{H} / \mathrm{M}_{4 \mathrm{~h}}$ values in relation to the adopted lower limit $\left(H / M_{4 h}=1.6\right)$ or taking the $H / M_{4 h}$ ratio as a continuous variable. The study confirmed that symptomatic patients with heart failure have worse prognosis with reduced myocardial I-123-MIBG uptake. The percentage of cardiac events within the observation period of 2 years was $15 \%$ at $H / M_{4 h} \geq 1.6$ and was significantly greater at $\mathrm{H} / \mathrm{M}_{4 \mathrm{~h}}<1.6$ (37\%). The ADMIRE-HF study confirmed the independent prognostic value of I-123-MIBG scintigraphy for the assessment of patients with heart failure.

The main cause of death in the case of HF patients is cardiac death due to ventricular disorders of heart rate. In the case of some patients the final arrhythmia is a natural result of the end-stage irreversible heart pump dysfunction; while in the case of some patients functioning quite well, sudden cardiac death (SCD) occurs. It was suggested in numerous studies that the disorders of MIBG uptake can be used as predictors of the higher risk of serious ventricular arrhythmias and SCD [43,70-73]. On the other hand, a high level of myocardial MIBG uptake, measured by the $\mathrm{H} / \mathrm{M}$ ratio, has a high value as a negative predictor, especially for potentially life-threatening arrhythmias. Thus, the results of extended MIBG studies can be used in the future for optimising therapy for HF patients, in particular when considering very expensive therapies involving specialist devices, such as implantable cardioverter 
defibrillator (ICD), cardiac resynchronisation therapy (CRT), left ventricular assist device (LVAD) [73, 74].

I-123-MIBG studies can also be used for monitoring the efficacy of these therapies. The recently published study of Drakos et al. [75], in which patients at the end-stage of HF and provided with LVAD were described, showed that the clinical, functional and haemodynamic improvement observed after 3 months was associated with better MIBG uptake and washout parameters in scintigraphic imaging. Another recently published study showed that the reduced $\mathrm{H} / \mathrm{M}$ ratio was associated with weak response to the applied cardiac resynchronisation therapy [76] .

\section{I-123-MIBG myocardial imaging in neurological pathological conditions}

Since 1994 there have been published papers describing the application of MIBG myocardial scintigraphy as a diagnostic and potentially prognostic tool in neurological pathological conditions, mainly Parkinson's disease.

A distinctive objective of neuroimaging techniques is to enable differentiating between neurodegenerative diseases, in particular diseases with Lewy bodies — including Parkinson's disease (PD) or dementia with Lewy bodies (DLB) - and Parkinson's syndromes or other types of dementia [including Alzheimer's disease (AD)] on account of the significant differences in their treatment. The accuracy of clinical diagnostic criteria for these diseases is limited; the similarity between specific clinical symptoms can delay diagnosis and application of treatment.

In 1994 Hakusui et al. [77] described a total lack of myocardial MIBG accumulation in patients with Parkinson's disease. Five years later Satoh et al. [78] demonstrated not so much non-existence of uptake as substantially reduced uptake, based on the H/M ratio, in patients with Parkinson's disease compared to healthy subjects - which was confirmed in the subsequent studies.

Recently Muxi et al. have shown that the significantly reduced $\mathrm{H} / \mathrm{M}_{4 \mathrm{~h}}$ ratio is a scintigraphic parameter which best differentiates between PD patients and healthy people and that the optimised threshold value of 1.43 detects PD with sensitivity at $93 \%$ and specificity at $100 \%$ [40].

Sawada et al. have assessed the diagnostic accuracy of I-123-MIBG scintigrams in detecting Parkinson's disease in a large group (400 patients) suspected of this disease [79]. In 267 patients the disease was diagnosed based on diagnostic criteria and confirmed based on magnetic resonance results. The myocardial MIBG accumulation was significantly reduced in PD patients $\left(H / M_{20 m}\right.$ was 1.66 vs. 2.39 in non-PD patients, and the $H / M_{4 h}$ ratio was 1.44 vs. 2.42). With an optimised cut-off threshold at 1.68 for $\mathrm{H} / \mathrm{M}_{4 h}$, the sensitivity of PD detection was $84 \%$ and specificity $90 \%$. However, in a subgroup of patients diagnosed with the disease not more than 3 years earlier, the sensitivity of PD detection was $73 \%$ and specificity $88 \%$ with an optimised cut-off threshold at 1.8 for $\mathrm{H} / \mathrm{M}_{4 \mathrm{~h}}$. The authors determined that the diagnostic accuracy of MIBG myocardial scintigraphy is limited in the case of patients with early-stage PD due to the insufficient sensitivity.

Similarly, the study of Ishibashi et al., in which PD was diagnosed based on clinical criteria and PET dopamine tests, and compared to Parkinson's syndromes (non-PD), showed that
MIBG scintigraphy has a limited diagnostic value for patients with early-stage PD. In a general group of PD patients, with an optimised cut-off threshold for $\mathrm{H} / \mathrm{M}_{4 \mathrm{~h}}$ at 1.60 , the sensitivity was determined at $71 \%$ and specificity at $93 \%$ for the disease detection, but in a subgroup of patients with early-stage disease, the sensitivity was only $54 \%$ [80].

The reduced MIBG uptake and the corresponding degeneration of the myocardial sympathetic innervation was observed in early stages of DLB and PD by Suzuki et al., and the functions of the adrenergic system, measured with the $\mathrm{H} / \mathrm{M}$ ratio, was significantly reduced in DLB patients compared to PD patients [81].

In the differentiation between DLB and Alzheimer's disease it was shown that the $\mathrm{H} / \mathrm{M}$ ratio is significantly lower in DLB patients $[82,83]$. In the studies of Yoshita et al. the optimised threshold value at 1.68 for $\mathrm{H} / \mathrm{M}_{4 \mathrm{~h}}$ enabled $100 \%$ sensitivity and specificity of the differentiation process; it was also shown that the WR value at $23.6 \%$ enables differentiating between DLB (with greater washout rate) and Alzheimer's disease with sensitivity at $84 \%$ and specificity at $83 \%$ [83].

Estorch et al. investigated the possibility of early MIBG-based diagnostic differentiation between DLB and other neurodegenerative diseases characterised by cognitive disorders [84]. The results of MIBG-based examinations at an early stage of the disease were verified based on the final clinical diagnosis made 4 years later, after the disease developed. The H/M ratio was significantly reduced in patients diagnosed with DLB in relation to patients with other neurodegenerative diseases. The $H / M_{4 h}$ ratio at 1.36 enabled differentiating between DLB and other diseases with sensitivity at $94 \%$ and specificity at $96 \%$, while the WR value was significantly higher in DLB patients only compared to patients with Alzheimer's disease (34\% vs. 22\%).

\section{Conclusions}

Although first introduced as a cardiac diagnostic procedure 30 years ago, l-123-MIBG myocardial scintigraphic methods continue to be mostly applied in scientific research. Their advantage over the existing diagnostic methods has not been sufficiently documented (lack of multi-centre randomised studies with large groups of patients), even though there are numerous reports of the diagnostic and/or prognostic importance of this examination, especially in the case of heart failure. The testing results of MIBG-based examinations have been described for a variety of heart diseases, including: primary arrhythmia, myocardial ischaemia, cardiomyopathies, myocarditis, diabetic neuropathy, in relation to the assessment of the development of the innervation system after heart transplant, assessment of cardiotoxicity of anticancer chemotherapy, monitoring of the therapy effects, and in neurological diseases (mainly Parkinson's disease).

The paper discusses in more detail the testing results in some clinical situations which have been described in a number of publications and new original papers over the past 2 years, demonstrating the continuous/growing interest in I-123-MIBG myocardial examinations.

The results of two extensive retrospective works and a major prospective, multi-centre international ADMIRE-HF study, published in the recent years, confirmed that I-123-MIBG scintigraphy 
has a high independent prognostic value for the assessment of patients suffering from heart failure: patients with the lowest MIBG uptake are patients with the worst prognosis. Improper myocardial I-123-MIBG uptake can be indicative of the increased risk of ventricular arrhythmia and SCD in patients with heart failure.

I-123-MIBG myocardial scintigraphy seems also to have a considerable potential for detection and differentiation of neurodegenerative diseases. In the case of diseases with Lewy bodies, such as Parkinson's disease and dementia with Lewy bodies, the functions of the adrenergic system are to a significant extent worse compared to the healthy population and patients with Parkinson's syndromes or other types of dementia. Furthermore, the functions of the cardiac adrenergic system are significantly worse in patients suffering from dementia with Lewy bodies compared to patients with Parkinson's disease, Alzheimer's disease or other neurodegenerative diseases characterised by cognitive disorders. However, the sensitivity of MIBG-based myocardial scintigraphy in detecting early stage of Parkinson's disease seems to be limited.

\section{References}

1. Kline RC, Swanson DP, Wieland DM et al. Myocardial imaging in man with I-123 mataiodobenzylguanidine. J Nucl Med 1981; 22: 129-132.

2. Sisson JC. The adrenergic nervous system of the heart and nuclear medicine. In: Freeman LM, Editor. Nuclear Medicine Annual 1993. Raven Press, New York 1993: 233-257.

3. Schafers M, Schober O, Lerch H. Cardiac sympathetic neurotransmission scintigraphy. Eur J Nucl Med 1998; 25: 435-441.

4. Matsunari I, Wieland D, Schweiger M. Neurotransmitter imaging. In: Zaret BL, Beller GA, Editors. Nuclear cardiology. State of the art and future directions. 2nd ed. Mosby, St. Louis, Missouri 1999: 587-600.

5. Hattori N, Schweiger M. Metaiodobenzylguanidine scintigraphy of the heart: what have we learnt clinically? Eur J Nucl Med 2000; 27: 1-6.

6. Flotats A, Carrio I. Cardiac neurotransmission SPECT imaging. J Nucl Cardiol 2004; 11: 587-602.

7. Bengel FM, Schweiger M. Assessment of cardiac sympathetic neuronal function using PET imaging. J Nucl Cardiol 2004; 11: 603-613.

8. Yu M, Guaraldi M, Bozek J et al. LMI1195: A new 18F benzylguanidine analog for PET cardiac sympathetic neuronal imaging [Abstract]. J Am Colll Cardiol 2010; 55: A88.

9. Merlet P, Delforge J, Syrota A et al. Positron emission tomography with 11C CGP-12177 to assess beta-adrenergic receptor concentration in idiopathic dilated cardiomyopathy. Circulation 1993; 87: 1169-1178.

10. Caldwell JH, Link JM, Levy WC et al. Evidence for pre- to postsynaptic mismatch of the cardiac sympathetic nervous system in ischemic congestive heart failure. J Nucl Med 2008; 49: 234-241.

11. Glowniak JV, Turner FE, Gray LL et al. lodine-123 metaiodobenzylguanidine imaging of the heart in idiopathic congestive cardiomyopathy and cardiac transplants. J Nucl Med 1989; 30: 1182-1191.

12. Dae MW, De Marco T, Botvinic EH et al. Scintigraphic assessment of MIBG uptake in globally denervated human and canine hearts _ implications for clinical studies. J Nucl Med 1992; 33: 1444-1450.

13. Schofer J, Spielmann R, Schuchert A et al. lodine-123 meta-iodobenzylguanidine scintigraphy: a noninvasive method to demonstrate myocardial adrenergic nervous system disintegrity in patients with idiopathic dilated cardiomyopathy. J Am Coll Cardiol 1988; 12: 1252-1258.

14. Stanton MS, Tuli MM, Radtke NL et al. Regional sympathetic denervation after myocardial infarction in humans detected noninvasively using I-123-Metaiodobenzylguanidine. J Am Coll Cardiol 1989; 14: 1519-1526.
15. Dae MW, Herre JM, O'Connell JW et al. Scintigraphic assessment of sympathetic innervation after transmural versus nontransmural myocardial infarction. J Am Coll Cardiol 1991; 17: 1416-1423.

16. McGhie Al, Corbett JR, Akers MS et al. Regional cardiac adrenergic function using I-123 meta-iodobenzylguanidine tomographic imaging after acute myocardial infarction. Am J Cardiol 1991; 67: 236-242.

17. Simula S, Lakka T, Laitinen $T$ et al. Cardiac adrenergic denervation in patients with non-Q-wave versus Q-wave myocardial infarction. Eur J Nucl Med 2000; 27: 816-821.

18. Arbab AS, Koizumi $K$, Takano $\mathrm{H}$ et al. Parameters of dynamic and static iodine-123-MIBG cardiac imaging. J Nucl Med 1995; 36: 962-968.

19. Nakajo M, Shimabukuro K, Yoshimura $\mathrm{H}$ et al. lodine-131 metaiodobenzylguanidine intra- and extravesicular accumulation in the rat heart. J Nucl Med 1986; 27: 84-89.

20. Frahati J, Bier D, Scheubeck $M$ et al. Effect of specific activity on cardiac uptake of iodine-123-MIBG. J Nucl Med 1997; 38: 447-451.

21. Solanki KK, Bomanji J, Moyes $\mathrm{J}$ et al. A pharmacological guide to medicines which interfere with biodistribution of radiolabelled meta-iodobenzylguanidine (MIBG). Nucl Med Commun 1992; 13: 513-521.

22. Mayer $\mathrm{S}$, Karanikas $\mathrm{G}$, Rodrigues $\mathrm{M}$, Sinzinger $\mathrm{H}$. Influence of drugs on myocardial iodine-123 metaiodobenzylguanidine uptake in rabbit myocardium. Eur J Nucl Med 2000; 27: 340-345.

23. Olivier $P$, Colarinha $P$, Fettich $J$ et al. Guidelines for radioiodinated MIBG scintigraphy in children. Eur J Nucl Med Mol Imaging 2003; 30: BP45-BP50.

24. Bombardieri E, Giammarile F, Aktolun C et al. 1311/123I-Metaiodobenzylguanidine ( $\mathrm{mlBG}$ ) scintigraphy — procedures guidelines for tumour imaging 2010; www.eanm.org.

25. Flotats A, Carrio I, Agostini D et al. Proposal for standardization of 123I-metaiodobenzylguanidine (MIBG) cardiac sympathetic imaging by the EANM Cardiovascular Committee and the European Council of Nuclear Cardiology. Eur J Nucl Med Mol Imaging 2010; 37: 1802-1812.

26. Jacobson AF, Senior R, Cerqueira MD et al. Myocardial iodine-123 meta-iodobenzylguanidine imaging and cardiac events in heart failure. Results of the prospective ADMIRE-HF (AdreView Myocardial Imaging for Risk Evaluation in Heart Failure) Study. J Am Coll Cardiol 2010; 55: 2212-2221.

27. Agostini D, Verberne HJ, Burchert W et al. I-123-mIBG myocardial imaging for assessment of risk for a major cardiac event in heart failure patients: insights from a retrospective European multicenter study. Eur J Nucl Med Mol Imaging 2008; 35: 535-546.

28. Verberne HJ, Brewster LM, Somsen GA, van Eck-Smit BLF. Prognostic value of myocardial 123I-metaiodobenzylguanidine (MIBG) parameters in patients with heart failure: a systematic review. Eur Heart J 2008; 29: 1147-1159.

29. Nakajima K, Taki J, Tonami N, Hisada K. Decreased ${ }^{123}$-MIBG uptake and increased clearance in various cardiac diseases. Nucl Med Commun 1994; 15: 317-323.

30. Richalet JP, Merlet P, Bourguignon M et al. MIBG scintigraphic assessment of cardiac adrenergic activity in response to altitute hypoxia. J Nucl Med 1990; 31: 34-37.

31. Merlet P, Valette H, Dubois-Rande JL et al. Prognostic value of cardiac metaiodobenzylguanidine imaging in patients with heart failure. J Nucl Med 1992; 33: 471-477.

32. Verberne HJ, Habraken JBA, van Eck-Smit BLF. Variations in 123I-metaiodobenzylguanidine (MIBG) late heart mediastinal ratios in chronic heart failure: a need for standardisation and validation. Eur J Nucl Med Mol Imaging 2008; 35: 547-553.

33. Agostini D, Carrio I, Verberne HJ. How to use myocardial 123I-MIBG scintigraphy in chronic heart failure Eur $\mathrm{J}$ Nucl Med Mol Imaging 2009; 36: 555-559. 
34. Gill JS, Hunter GJ, Gane J et al. Asymmetry of cardiac [123|] meta-iodobenzyl-guanidine scans in patients with ventricular tachycardia and a"clinicall normal" heart. Br Heart J 1993; 69: 6-13.

35. Sisson JC, Shapiro B, Mallette S et al. Mataiodobenzylguanidine to map scintigraphically the adrenergic nervous system in man. J Nucl Med 1987; 28: 1625-1633.

36. Gill JS, Hunter GJ, Gane G, Camm AJ. Heterogenity of the human myocardial sympathetic innervation: In vivo demonstration by iodine 123-labeled metaiodobenzylguanidine scintigraphy. Am Heart J 1993; 126: 390-398.

37. Tsuchimochi S, Tamaki N, Tadamura E et al. Age and gender differences in normal myocardial adrenergic neuronal function evaluated by iodine-123-MIBG imaging. J Nucl Med 1995; 36: 969-974

38. Morozumi T, Kusuoka H, Tani A et al. Myocardial iodine-123-metaiodobenzylguanidine images and autonomic nerve activity in normal subjects. J Nucl Med 1997; 38: 49-52.

39. Teresińska A, Śliwiński M, Konieczna S et al. Changes in cardiac adrenergic nervous system after transmyocardial laser revascularisation assessed by I-123-MIBG SPECT. A preliminary report. Kardiol Pol 2004; 60: 15-20.

40. Muxí A, Paredes P, Navales I et al. Diagnostic cutoff points for 123I-MIBG myocardial scintigraphy in a Caucasian population with Parkinson's disease. Eur J Nucl Med Mol Imaging 2011; 38: 1139-1146.

41. Mitrani RD, Klein LS, Miles WM et al. Regional cardiac sympathetic denervation in patients with ventricular tachycardia in the absence of coronary artery disease. J Am Coll Cardiol 1993; 22: 1344-1353.

42. Wichter $\mathrm{T}$, Lerch $\mathrm{H}$, Schafers $M$ et al. Regional myocardial sympathetic dysinnervation in arrhythmogenic right ventricular cardiomyopathy. An analysis using 123I-meta-lodobenzyloguanidine scintigraphy. Circulation 1994; 89: 667-683.

43. Akutsu $Y$, Kaneko K, Kodama $Y$ et al. Cardiac sympathetic nerve abnormality predicts ventricular tachyarrhythmic events in patients without conventional risk of sudden death. Eur J Nucl Med Mol Imaging 2008; 35: 2066-2073.

44. Matsuo S, Takahashi M, Nakamura Y, Kinoshita M. Evaluation of cardiac sympathetic innervation with iodine-123-metaiodobenzylguanidine imaging in silent myocardial ischemia. J Nucl Med 1996; 37: 712-717.

45. Sakata K, Yoshida H, Hoshino T, Kurata C. Sympathetic nerve activity in the spasm-induced coronary artery region is associated with disease activity of vasospastic angina. J Am Coll Cardiol 1996; 28: 460-464.

46. Sakata K, Shirotani M, Yoshida H, Kurata C. Iodine-123 metaiodobenzylguanidine cardiac imaging to identify and localize vasospastic angina without significant coronary artery narrowing. J Am Coll Cardiol 1997; 30: 370-376.

47. Lanza GA, Giordano A, Pristipino Ch et al. Abnormal cardiac adrenergic nerve function in patients with syndrome $\mathrm{X}$ detected by 123 | metaiodobenzylguanidine myocardial scintigraphy. Circulation 1997; 96: 821-826.

48. Taki J, Yasuhara S, Takamatsu T et al. Value of iodine-123 metaiodobenzylguanidine scintigraphy in patients with vasospastic angina. Eur J Nucl Med 1998; 25: 229-234

49. DeMarco T, Dae M, Yuen-Green MS et al. lodine-123 metaiodobenzylguanidine scintigraphic assessment of the transplanted human heart: evidence for late reinnervation. J Am Coll Cardiol 1995; 25: 927-931.

50. Carrio I, Estorch M, Berna L et al. Indium-11-antimyosin and iodine-123-MIBG studies in early assessment of doxorubicin cardiomyopathy. J Nucl Med 1995; 36: 2044-2049.

51. Lekakis J, Prassopoulos V, Athanassiadis P et al. Doxorubicin-induced cardiac neurotoxicity: study with iodine-123-labeled metaiodobenzylguanidine scintigraphy. J Nucl Cardiol 1996; 3: 37-46.

52. Olmos RA, ten Bokkel Huinink WW, ten Hoeve RFA et al. Assessment of anthracycline-related myocardial adrenergic derangement by [123I] MIBG scintigraphy. Eur J Cancer 1995; 31A: 26-31.
53. Cohen-Solal A, Rouzet F, Berdeaux A et al. Effects of carvedilol on myocardial sympathetic innervation in patients with chronic heart failure. J Nucl Med 2005; 46: 1796-1803.

54. Henderson EB, Kahn JK, Corbett JR et al. Abnormal I-123 metaiodobenzylguanidine myocardial washout and distribution may reflect myocardial adrenergic derangement in patients with congestive cardiomyopathy. Circulation 1988; 78: 1192-1199.

55. Brush JE, Eisenhofer G, Garty M et al. Cardiac norepnephrine kinetics in hypertrophic cardiomyopathy. Circulation 1989; 79: 836-844.

56. Nakajima K, Bunko H, Taki J et al. Quantitative analysis of 123l-metaiodobenzylguanidine (MIBG) uptake in hypertrophic cardiomyopathy. Am Heart J 1990; 119: 1329-1337.

57. Suwa M, Otake Y, Moriguchi A et al. lodine-123 metaiodobenzylguanidine myocardial scintigraphy for prediction of response to beta-blocker therapy in patients with dilated cardiomyopathy. Am Heart J 1997; 133: 353-358.

58. Merlet P, Benvenuti C, Moyse D et al. Prognostic value of MIBG imaging in idiopathic dilated cardiomyopathy. J Nucl Med 1999; 40: 917-923.

59. Agostini D, Babatasi G, Manrique A et al. Impairment of cardiac neuronal function in acute myocarditis: lodine-123-MIBG scintigraphy study. J Nucl Med 1998; 39: 1841-1844.

60. Mantysaari M, Kuikka J, Mustonen $\mathrm{J}$ et al. Noninvasive detection of cardiac sympathetic nervous dysfunction in diabetic patients using [123I] metaiodobenzylguanidine. Diabetes 1992; 41: 1069-1075.

61. Langer A, Freeman MR, Josse RG, Armstrong PW. Metaiodobenzylguanidine imaging in diabetes mellitus: assessment of cardiac sympathetic denervation and its relation to autonomic dysfunction and silent myocardial ischemia. J Am Coll Cardiol 1995; 25: 610-618.

62. Hattori N, Tamaki N, Hayashi T et al. Regional abnormality of iodine-123-MIBG in diabetic hearts. J Nucl Med 1996; 37: 1985-1990.

63. Nagamachi S, Fujita S, Nishii R et al. Prognostic value of cardiac l-123 metaiodobenzylguanidine imaging in patients with noninsulin-dependent diabetes mellitus. J Nucl Cardiol 2006; 13: 34-42.

64. Muxi A, Magrina J, Martin F et al. 99mTc Tetrofosmin and 123I-MIBG scintigraphy in the assessment of transmyocardial laser revascularisation (Abstract). J Nucl Cardiol 2001; 8: S22.

65. Teresińska A, Śliwiński M, Konieczna S et al. Assessment of cardiac adrenergic nervous system with I-123-MIBG SPECT in patients submitted to transmyocardial laser revascularisation. Preliminary data (Abstract). Eur J Nucl Med 2001; 28: 1206.

66. Muxi A, Magrina J, Martin F et al. Technetium 99m-labeled tetrofosmin and iodine 123-labeled metaiodobenzylguanidine scintigraphy in the assessment of transmyocardial laser revascularisation. J Thorac Cardiov Surg 2003; 125: 1493-1498.

67. Teresińska A. Radioisotopic assessment of the influence of transmyocardial laser revascularisation (TMLR) on perfusion and innervation of left ventricular myocardium. Habilitation dissertation. Oficyna Wydawnicza Akademii Medycznej, Warszawa, Zam. 415/2007.

68. Horiguchi Y, Morita Y, Tsurikisawa N, Akiyama K. 123I-MIBG imaging detects cardiac involvement and predicts cardiac events in Churg-Strauss syndrome. Eur J Nucl Med Mol Imaging 2011; 38: 221-229.

69. Dickstein K, Cohen-Solal A, Filippatos G et al. ESC Guidelines for the diagnosis and treatment of acute and chronic heart failure 2008. Eur Heart J 2008; 29: 2388-2442.

70. Yamada T, Shimonagata T, Fukunami $\mathrm{N}$ et al. Comparison of the prognostic value of cardiac iodine-123 metaiodobenzylguanidine imaging and heart rate variability in patients with chronic heart failure. A prospective study. J Am Coll Cardiol 2003; 41: 231-238.

71. Kioka H, Yamada T, Mine T et al. Prediction of sudden death in patients with mild-to-moderate chronic heart failure by using cardiac iodine-123 metaiodobenzylguanidine imaging. Heart 2007; 93: 1213-1218.

72. Tamaki S, Yamada T, Okuyama Y et al. Cardiac iodine-123 metaiodobenzylguanidine imaging predicts sudden cardiac death independently of left ventricular ejection fraction in patients with chronic heart 
failure and left ventricular systolic dysfunction: results from a comparative study with signal averaged electrocardiogram, heart rate variability, and QT dispersion. J Am Coll Cardiol 2009; 53: 426-435.

73. Boogers MJ, Borleffs CJ, Henneman MM et al. Cardiac sympathetic denervation assessed with 123-iodine metaiodobenzylguanidine imaging predicts ventricular arrhythmias in implantable cardioverter-defibrillator patients. J Am Coll Cardiol 2010; 55: 2769-2777.

74. Arora R, Ferrick KJ, Nakata T et al. I-123 MIBG imaging and heart rate variability analysis to predict the need for an implantable cardioverter defibrillator. J Nucl Cardiol 2003; 10: 121-131.

75. Drakos SG, Athanasoulis T, Malliaras KG et al. Myocardial sympathetic innervation and long-term left ventricular mechanical unloading. J Am Coll Cardiol Imag 2010; 3: 64-70.

76. D'Orio Nishioka SA, Filho MM, Soares Brandao SC et al. Cardiac sympathetic activity pre and post resynchronization therapy evaluated by 123I-MIBG myocardial scintigraphy. J Nucl Cardiol 2007; 14: 852-859.

77. Hakusui S, Yasuda T, Yanagi T et al. 123I-MIBG myocardial scintigraphical analysis in patients with and without autonomic disorder. Clin Neurol 1994; 34: 402-404.

78. Satoh A, Serita T, Seto M et al. Loss of 123I-MIBG uptake by the heart in Parkinson's disease: assessment of cardiac sympathetic denervation and diagnostic value. J Nucl Med 1999; 40: 371-375
79. Sawada H, Oeda T, Yamamoto K et al. Diagnostic accuracy of cardiac metaiodobenzylguanidine scintigraphy in Parkinson disease. Eur J Neurol 2009; 16: 174-182.

80. Ishibashi K, Saito Y, Murayama S et al. Validation of cardiac (123) I-MIBG scintigraphy in patients with Parkinson's disease who were diagnosed with dopamine PET. Eur J Nucl Med Mol Imaging 2010; 37: 3-11.

81. Suzuki M, Kurita A, Hashimoto M et al. Impaired myocardial 123I-metaiodobenzylguanidine uptake in Lewy body disease: comparison between dementia with Lewy bodies and Parkinson's disease. J Neurol Sci 2006; 240: 15-19.

82. Yoshita M, Taki J, Yokoyama K et al. Value of 123I-MIBG radioactivity in the differential diagnosis of DLB from AD. Neurology 2006; 66: 1850-1854.

83. Wada-Isoe K, Kitayama M, Nakaso K, Nakashima K. Diagnostic markers for diagnosing dementia with Lewy bodies: CSF and MIBG cardiac scintigraphy study. J Neurol Sci 2007; 260: 33-37.

84. Estorch M, Camacho V, Paredes P et al. Cardiac (123)I-metaiodobenzylguanidine imaging allows early identification of dementia with Lewy bodies during life. Eur J Nucl Med Mol Imaging 2008; 35: 1636-1641. 